\title{
Vancomycin-resistant Enterococcus faecium Meningitis
}

The Editor,

Sir,

A 19-year old male who was operated on due to a suprasellar mass and hydrocephaly was observed to develop fever, headache, nausea and vomiting on the $5^{\text {th }}$ postoperative day. During the physical examination, the patient had nuchal rigidity but the other systemic results were normal. The patient's leukocyte count was $14.600 / \mathrm{mm}^{3}$, sedimentation rate was 32 $\mathrm{mm} / \mathrm{h}$, and his C-reactive protein (CRP) was $7.9 \mathrm{mg} / \mathrm{dL}$. Based on the pre-diagnosis of meningitis, the patient underwent a lumbar puncture. During the analysis, cerebrospinal fluid (CSF) was clear with increased pressure, and the test results indicated the leukocyte count as $200 / \mathrm{mm}^{3}$ in the CSF. The patient in the CSF was diagnosed with nosocomial meningitis and was started on a regimen with meropenem and vancomycin.

As the high-fever, headache and nuchal rigidity continued on the $15^{\text {th }}$ day of the treatment, the patient underwent a second CSF analysis. This analysis gave the following results: leukocyte count: $4160 / \mathrm{mm}^{3}$, the gram staining showed grampositive cocci and vancomycin-resistant enterococcus faecium (VREF) growth was observed in the CSF culture. The meropenem and vancomycin treatment was discontinued and the patient was started on a regimen with linezolid and daptomycin. Also, the patient's rectal smear culture resulted in VREF growth. On the $3^{\text {rd }}$ day of the therapy, the patient was placed an external ventricular drain catheter due to hydrocephaly.

On the $5^{\text {th }}$ day of the therapy, the patient's general condition improved, his fever responded to the treatment, his symptoms diminished and no cells were observed in the CSF follow-up test. When the fever and headache recurred on the $14^{\text {th }}$ day of the linezolid and daptomycin therapy, the patient was applied a ventriculoperitoneal shunt. In the repeated analysis, the CSF was turbid with increased pressure, and the test results indicated the leukocyte count as $1750 / \mathrm{mm}^{3}$. Based on the diagnoses of non-VRE meningitis, meropenem was added to the linezolid therapy and the daptomycin treatment was discontinued on the $14^{\text {th }}$ day. When the CFS culture showed enterobacter aerogenes growth, the meropenem therapy was continued. After the patient's clinical, laboratory and CSF findings were improved, the meropenem therapy was continued until the $14^{\text {th }}$ day and the linezolid therapy was continued until the $28^{\text {th }}$ day when the patient was discharged.

Due to their intrinsic resistance against various antibiotics, ability to gain resistance through genetic materials, and the associated increase in vancomycin resistance in recent years, enterococci constitute a serious cause of nosocomial infections (1). The majority of the enterococcal meningitis cases are caused by enterococcus faecalis, while enterococcus fae- cium is responsible for $10 \%$ of the cases. However, the increase in the ratio of ampicillin and VREF causes serious concerns in terms of treatment (2).

Vancomycin-resistant enterococcus is a rare cause of meningitis which may be associated with head trauma, shunts or CSF leaks and leads to high mortality rates (3). Since adult cases of VRE meningitis are very rare, the clinical data on antibiotic treatment is limited to the reported patients. A number of antibiotics including teicoplanin, chloramphenicol, rifampicin, clindamycin, penicillin, quinupristin-dalfopristin, linezolid, daptomycin and tigecycline have been used in various combinations in the treatment of VREF meningitis (4). While the cure rates were lower with the antibiotics used in the treatment of VREF meningitis reported in previous years, the response rates to the treatment were observed to increase in the last decade, especially after the use of antibiotics such as, linezolid and daptomycin effective against $\operatorname{VRE}(4,5)$.

In the light of the recently reported data, there are no ideal treatment against VREF meningitis. However, linezolid monotherapy or the combination of daptomycin and linezolid seem to be the primary alternative with high cure rates.

Keywords: Meningitis, vancomycin-resistant enterococcus faecium

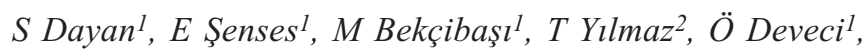
R Tekin ${ }^{1}$

From: ${ }^{1}$ Department of Infectious Diseases and Clinical Microbiology, ${ }^{2}$ Department of Neurosurgery, Faculty of Medicine, Dicle University, Diyarbakır, Turkey.

Correspondence: M Bekçibaşl, Department of Infectious Diseases and Clinical Microbiology, Faculty of Medicine, Dicle University, Diyarbakır, Turkey. Fax: +4122488440 , e-mail: m_bekcibasi@hotmail.com

\section{REFERENCES}

1. Huycke MM, Sahm DF, Gilmore MS. Multiple-drug resistant enterococci: the nature of the problem and an agenda for the future. Emerg Infect Dis 1998; 4: 239-49.

2. Zeana C, Kubin CJ, Della-Latta P, Hammer SM. Vancomycin-resistant Enterococcus faecium meningitis successfully managed with linezolid: case report and review of the literature. Clinical infectious diseases: an official publication of the Infectious Diseases Society of America 2001; 33: 477-82.

3. Pintado V, Cabellos C, Moreno S, Meseguer MA, Ayats J, Viladrich PF. Enterococcal meningitis: a clinical study of 39 cases and review of the literature. Medicine (Baltimore) 2003; 82: 346-64.

4. Knoll BM, Hellmann M, Kotton CN. Vancomycin-resistant Enterococcus faecium meningitis in adults: case series and review of the literature. Scand J Infect Dis 2013; 45: 131-9.

5. Duygu F, Balcı PÖ, Solmaz M, Uçar NS. Meningitis associated with Vancomycin resistant Enterococcus casseliflavus: First report. J Microbiol Infect Dis. 2011; 1: 138-40. 